\title{
Consistent Multivariate Seasonal Adjustment for Gross Domestic Product and its Breakdown in Expenditures
}

\author{
Reinier Bikker ${ }^{1}$, Jan van den Brakel ${ }^{1}$, Sabine Krieg ${ }^{1}$, Pim Ouwehand ${ }^{1}$, \\ and Ronald van der Stegen ${ }^{1}$
}

\begin{abstract}
Seasonally adjusted series of Gross Domestic Product (GDP) and its breakdown in underlying categories or domains are generally not consistent with each other. Statistical differences between the total GDP and the sum of the underlying domains arise for two reasons. If series are expressed in constant prices, differences arise due to the process of chain linking. These differences increase if, in addition, a univariate seasonal adjustment, with for instance X-13ARIMA-SEATS, is applied to each series separately. In this article, we propose to model the series for total GDP and its breakdown in underlying domains in a multivariate structural time series model, with the restriction that the sum over the different time series components for the domains are equal to the corresponding values for the total GDP. In the proposed procedure, this approach is applied as a pretreatment to remove outliers, level shifts, seasonal breaks and calendar effects, while obeying the aforementioned consistency restrictions. Subsequently, X-13ARIMA-SEATS is used for seasonal adjustment. This reduces inconsistencies remarkably. Remaining inconsistencies due to seasonal adjustment are removed with a benchmarking procedure.
\end{abstract}

Key words: Seasonal adjustment; discrepancies; Kalman filter; multivariate structural time series models; X-13ARIMA-SEATS; benchmarking.

\section{Introduction}

Most national statistical institutes (NSIs) publish time series at an aggregated level and breakdowns in $K \geq 2$ domains, for instance the Gross Domestic Product (GDP) divided over industries or over expenditures.

It is common practice to adjust for seasonal and calendar effects. The latter are variations in time series that can be explained from variations in the calendar, such as working day patterns and national holidays. The aim of these adjustments is to make different reporting periods comparable. Seasonal and calendar adjustment procedures are generally based on univariate methods applied to the series of each publication domain separately. A consequence of such approaches is that adjusted figures at the aggregated level are not consistent with the sum of the adjusted figures of the underlying breakdown in $K$ publication domains. This is a well-known problem and the status quo is that no

\footnotetext{
${ }^{1}$ Statistics Netherlands, P.O. Box 4481, 6401CZ Heerlen, The Netherlands. Emails: r.bikker@cbs.nl, ja.vandenbrakel@cbs.nl, s.krieg@cbs.nl, p.ouwehand@cbs.nl, and rhm.vanderstegen@cbs.nl

Acknowledgments: The views expressed in this article are those of the authors and do not necessarily reflect the policy of Statistics Netherlands. The authors are grateful to the unknown referees, the associate editor, Harm Jan Boonstra (Statistics Netherlands) and Jan van Dalen (Statistics Netherlands) for reading and commenting on a former draft of this article.
} 
adequate solution exists. Eurostat's ESS guidelines on seasonal adjustment (Eurostat 2015) suggest computing the adjusted series at the aggregated level as a sum of the adjusted underlying domains, which is often referred to as the indirect approach. A drawback of this approach is that the most reliable estimates at the aggregated level are disregarded. Alternatively, if the discrepancies are small enough, they can be distributed by means of multivariate benchmarking techniques. These can be two-step procedures of benchmarking and reconciliation (Quenneville and Fortier 2006), or simultaneous methods as described in Di Fonzo and Marini (2011). In the Netherlands, however, the quarter-to-quarter changes in the discrepancies between the directly adjusted GDP and the sum of the adjusted series of its expenditures are often larger than the growth rate of GDP itself. This fact alone renders both remedies suggested by Eurostat unsuitable. For instance, in the first quarter after the 2013 recession, GDP grew by $0.1 \%$ if calculated directly, and by $-0.9 \%$ if calculated indirectly.

The purpose of this article is to develop a method that attempts to make consistent seasonal adjusted series using a multivariate structural time series modelling approach. We focus on GDP and a breakdown in different expenditures. However, the proposed method is general and can be applied in any situation where consistent seasonal and calendar adjustment is required.

Another discrepancy is introduced by chain linking (see Bloem et al. 2001), in which GDP and its expenditures are calculated as chain volumes. In chain linking, series of a constant price level are constructed by "chaining" volume growth rates. These volume growth rates are calculated by dividing the nominal growth rate by a price factor. As each of the series in the breakdown of GDP has its own price factor, discrepancies arise between the sum of the expenditures and GDP itself.

In the Dutch case, the discrepancies from chain linking are typically smaller than the discrepancies introduced by the adjustments for seasonal and calendar effects. Moreover, we noted that the size of the discrepancies due to seasonal and calendar adjustment grew larger in the period 2009-2013. This period is characterized by rapid changes in seasonal patterns following the financial crisis in 2008/2009. The increasing size of the discrepancies eventually lead to complaints from users. The discrepancies were noted in the press, and professional users also asked how to interpret our published results.

Besides discrepancies, there are more quality aspects related to seasonal adjustment. When new data points become available and are added to the series, better estimates of the trend, the seasonal and calendar effect of all previous quarters, can be made. Therefore, revisions are inherent to seasonal and calendar adjustment, which are acceptable, as long as their size is not excessive.

Traditionally, the quality of seasonal and calendar adjustment is assessed using a welldefined set of criteria. In the case of X-13ARIMA-SEATS (U.S. Census Bureau 2015), the method used at Statistics Netherlands, these are the Q- and M-diagnostics. They are numerical scores given to properties, such as the amount of seasonality compared to noise and the rate at which the seasonal component changes over time. These criteria are optimised for each time series individually. After performing seasonal and calendar adjustment, the resulting discrepancies are calculated and only when these are very large, the seasonal and calendar adjustment may be changed. Revisions are monitored, but 
never lead to changes in the setup of seasonal and calendar adjustment. So, the quality criteria that Statistics Netherlands traditionally applies, are (in order of decreasing importance):

1. Optimal quality diagnostics (specifically X-13ARIMA-SEATS's Q- and M-values).

2. Minimal statistical discrepancies between GDP and the sum of expenditures.

3. Minimal revision of the seasonal effect after adding new data points.

This is under the assumption that all criteria are within acceptable boundaries. As this was not the case in the Netherlands after the crisis in 2008/2009, the primary objective of the current research is to reduce the statistical discrepancy. This is achieved by introducing a multivariate approach. The consequence of the shift from an optimal univariate solution to a multivariate solution is that the seasonal and calendar adjustment of one series is influenced by another. Therefore, some aspects of the multivariate adjustment can be less optimal, when compared to the univariate case. However, a slightly lower quality (according to the Q- and M-diagnostics) can be equally acceptable for the users, as long as no residual seasonal effect can be found in a corrected series. Therefore, our goal is that, on average, the revisions and quality diagnostics should not deteriorate.

In this article, we describe two alternative approaches to adjustment for seasonal and calendar effects. The first approach applies a multivariate structural time series model to an aggregated series and its breakdown in $K$ subseries. The model estimates all components subject to the constraint that the sum of the subseries is equal to the components of the aggregated series. Unfortunately, the results of this approach are not satisfactory due to numerical problems. Furthermore, the estimates for the seasonal components are considered to be too volatile. Therefore, a second approach is developed, which is based on a combination of a multivariate structural time series model and routines of X-13ARIMA-SEATS. Under this approach, the discrepancies are sufficiently reduced, while the size of the revisions is in the same order as before.

In Section 2 we will first define the problem in a more precise way. In Section 3, we present the multivariate state-space method for consistent seasonal adjustment. Section 4 discusses the results and finally, the article closes with a conclusion in Section 5.

\section{Problem Definition}

Statistics Netherlands publishes quarterly figures for GDP with both the final expenditures and the value added by industry as domains. These breakdowns are called the expenditure approach and the production approach. Both breakdowns are computed in constant prices (chain linked volumes) and in current prices. In this article, we will focus on the expenditure approach in constant prices.

In this article the aggregate $B 1 G$, the GDP, is itemized in the subseries $P 7$ (imports), $P 3_{S 1 A}$ (consumption households), $P 3_{S 13}$ (consumption government), $P 51 G$ (gross fixed capital formation), $P 5 M$ (changes in stocks and inventories), $P 6$ (exports) and $S D$ (statistical discrepancy due to chain linking), that is,

$$
B 1 G=-P 7+P 3_{S 1 A}+P 3_{S 13}+P 51 G+P 5 M+P 6+S D .
$$


Statistics Netherlands publishes a very detailed tree-structured breakdown into expenditures, as explained in the Appendix (Section 6) on the breakdown in expenditures. The production approach is not considered in this article. Any breakdown of GDP has the same problems with additivity, so for brevity, we only use the above breakdown in this article.

The way we will handle the discrepancies arising from chain linking is by considering them as an extra subseries of the aggregate. It is a series that must be adjusted for seasonal and calendar effects, together with the other subseries of the aggregate. Therefore, the method we developed is also suitable for series where no chain linking takes place, such as current price data and any other set of series where preserving additivity, or at least reducing discrepancies due to adjustments for seasonal and calendar effects, is required.

As we will apply our model to chain volumes of GDP and its expenditures, the total statistical discrepancy after adjustments for seasonal and calendar effects can be divided in two parts, each with its own origin.

\subsection{Discrepancies Arising from Chain Linking}

The statistical discrepancies due to chain linking can be interpreted as the consequence of changes in relative prices of subseries of the aggregate. One can show that the sum of the chain linked expenditures is chain volume with different weights. In each link step, the chain volume of GDP is weighted with the relative values of its expenditures in the previous year, valued at prices of the previous year, whereas the sum of expenditures is weighted with the relative values of the previous year, in reference year prices. So, the statistical discrepancy due to chain linking is the difference between the value of the aggregate valued in previous year prices and the value of the aggregate valued in reference year prices. The discrepancies due to chain linking are therefore zero in the first year after the reference year and tend to be larger the further away they are from the reference year.

The statistical discrepancies due to chain linking typically have a slow moving long-term trendcycle, combined with a strong short-term pattern. The short-term pattern has a seasonal and an irregular component. Figure 1 shows a typical example for total GDP.

The statistical discrepancies due to chain linking are inherent to the way they are defined and should not be corrected, as this would harm the essence of a chain linked volume. The seasonal pattern of the discrepancies can be removed. In theory, the discrepancies due to chain linking could also show calendar effects. However, in the case of the Dutch economic series, they are negligibly small and we choose to ignore them.

The quarter-to-quarter changes of the seasonal adjusted GDP (GDP-SA) are very important results from the economic analysis. Therefore, it makes sense to also calculate the quarter-to-quarter changes of the statistical discrepancy and compare them to GDP-SA as follows:

$$
\% S D_{t}=\left(S D_{t}-S D_{t-1}\right) / B 1 G_{t-1} * 100 \%
$$




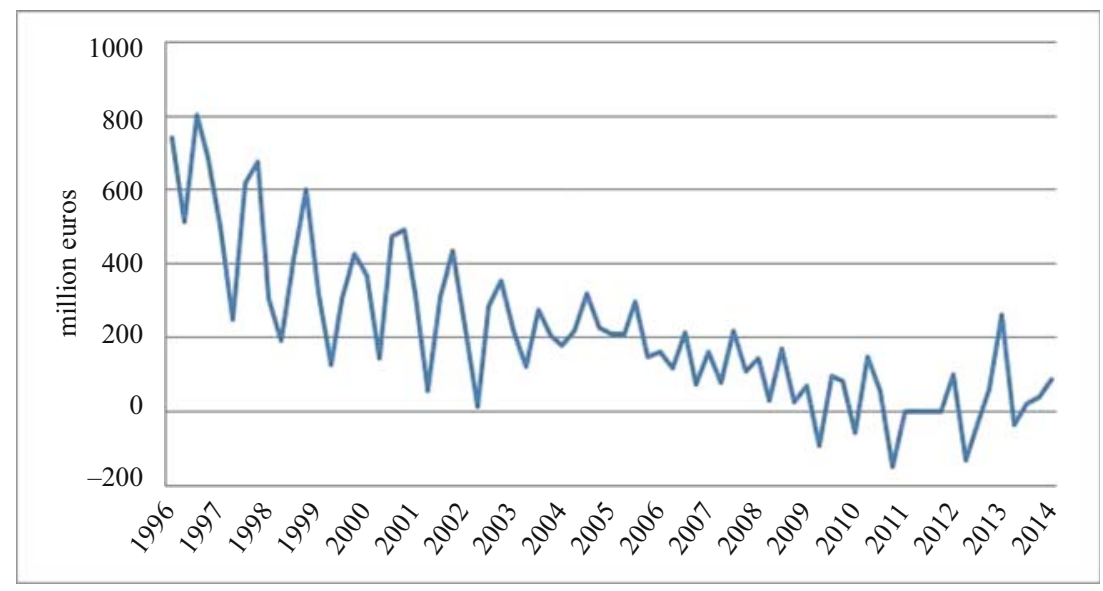

Fig. 1. Statistical discrepancies due to chain linking between Dutch GDP and the sum of the final expenditures (before adjustments for seasonal and calendar effects), reference year $=2010$.

When these quarter-to-quarter changes are of similar magnitude or larger than the changes of GDP-SA itself, the analysis of the latter, by breaking it down into components, is severely hampered.

Especially when looking at quarter-to-quarter changes, removing the seasonal pattern can lead to a large reduction in the size of the discrepancies from chain linking. This is shown in Figure 2, where the quarter-to-quarter changes of the discrepancies in percentages of GDP-SA have been adjusted for seasonal effects (i.e., the remaining series represents trend-cycle + irregular). The value of this series is mainly between $-0.1 \%$ and $0.1 \%$. To put this into perspective, the majority of GDP-SA growth rates are between $-0.5 \%$ and $0.5 \%$. As can be seen, the seasonal component is by far the largest component of the statistical discrepancy arising from chain linking. Therefore, with ideal seasonal and calendar adjustment, the adjusted statistical discrepancy should have a small influence on the interpretation of the economic growth and its components.

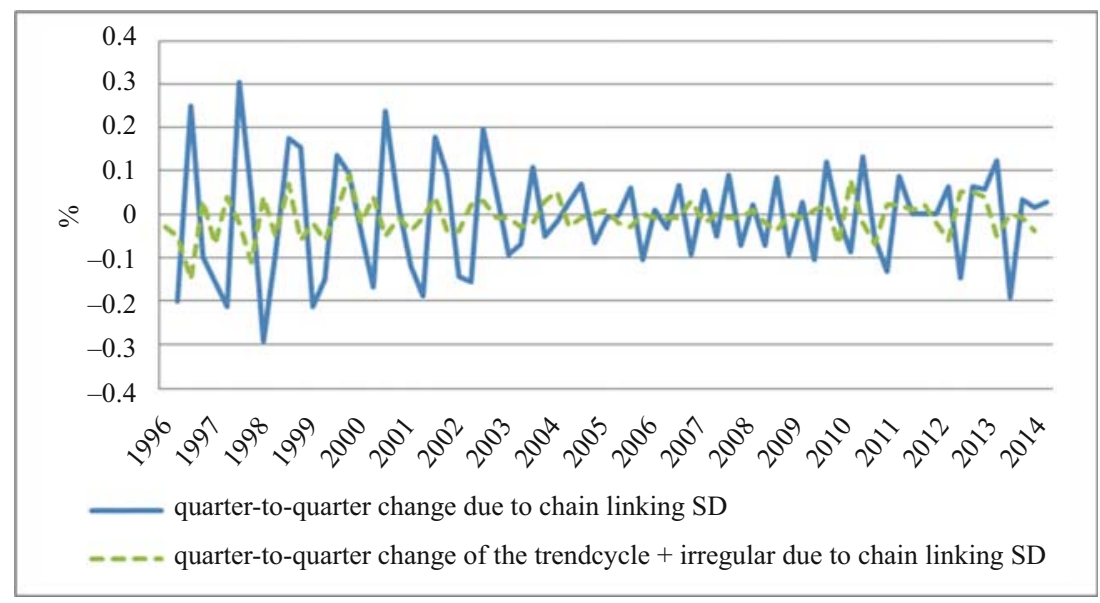

Fig. 2. Univariate seasonal correction of the discrepancies arising from chain linking. 


\subsection{Discrepancies from Adjustments for Seasonal and Calendar Effects}

The second part of the total statistical discrepancies is introduced by the estimation of seasonal and calendar effects. Seasonal and calendar adjustment assumes the following decomposition:

$$
y_{t}=L_{t}+S_{t}+\beta x_{t}+O L_{t}+S B_{t}+I_{t} .
$$

Here $y_{t}$ stands for any of the series appearing in Equation (1), $L$ denotes the trend-cycle, $S$ denotes the seasonal component, $\beta x$ denotes the regression component with $x$ as an auxiliary variable and $\beta$ as the regression coefficient, $O L$ denotes additive outliers and level shifts and $S B$ denotes seasonal breaks. Finally, $I$ is an irregular component for the unexplained variation. In this application, the regression component is used to adjust for calendar effects. In general, other regression effects can also be included, but this is not applied here. In a fully consistent adjustment, Equation (1) holds for each of the components in Equation (3). However, when these relations are not explicitly enforced, discrepancies will arise.

The process of seasonal and calendar adjustment consists of a pretreatment phase and the actual seasonal adjustment. In the pretreatment phase, we choose between multiplicative or additive adjustment. In the first case, the original series are logarithmically transformed before decomposition according to Equation (3) is computed. The other parts of the pretreatment phase are adjustments for calendar effects and other regression effects, removal of additive outliers, level shifts and seasonal breaks, and extrapolation of the series in order to apply symmetric filters. The actual seasonal adjustment consists in the application of seasonal and trend filters. In this phase, outlier detection takes place again. After seasonal and calendar adjustment, additive outliers and level shifts are reintroduced into the series. The final adjusted series is therefore equal to:

$$
y_{t}^{S A}=y_{t}-S_{t}-\beta x_{t}-S B_{t}=L_{t}+O L_{t}+I_{t} .
$$

All steps of the process may cause discrepancies:

Logarithmic transformation: Usually, this is done when this yields a better model fit, as, for instance, is current practice in X-13ARIMA-SEATS, see U.S. Census Bureau (2015). For some of our series, this would indeed be the preferred option. However, when multiplicative adjustment is chosen in at least one series, the logarithmic transformation can cause additional discrepancies.

Outlier detection: when each time series is analysed separately for significant outliers, discrepancies may arise when an outlier is significant in one series but not significant or even detectable in another. These may lead to relatively large incidental discrepancies. The outlier detection in both the pretreatment phase and the filtering phase can generate discrepancies. Here, the general term outlier is used for the combination of additive outliers, level shifts and seasonal breaks. A special case is the situation where the seasonal patterns change rapidly. In this case, discrepancies may arise around the period where the rapid change occurs, because these periods are considered to be outliers.

Calendar effects: Estimating the regression coefficients for the calendar effects for each series separately contributes to the discrepancies. The calendar effects in some series are not significantly different from zero (at a 5\% significance level). Therefore, it is not 
incorporated in the model of these series. This leads to relatively small discrepancies, evenly distributed along the length of the time series.

Extrapolations: The extrapolations are very sensitive to the model choice in X-13ARIMA-SEATS and to outliers at the beginning and end of the time series. This may lead to relatively large discrepancies at the beginning and end of the time series, and is a source of revisions.

Seasonal and trend filters: when different time series are treated with filters of a different length, which is usually the case, some discrepancies will arise in the seasonal components along the full length of the series.

The statistical discrepancy can be calculated by rewriting Equation (1):

$$
S D=B 1 G-\left(-P 7+P 3_{S 1 A}+P 3_{S 13}+P 51 G+P 5 M+P 6\right) .
$$

The right-hand side of this equation is called the indirect discrepancy, and the left hand side is called the direct discrepancy. This equation holds not only for the series itself, but in an ideal world, also for each of the components of Equation (3). However, due to the arguments mentioned above, this is not the case for the seasonal component, as is shown in Figure 3 for the period 1996-2014. The solid line is the indirect seasonal component from the chain linked index, calculated as the seasonal component of GDP minus the seasonal components of all other expenditures (right-hand side of Equation 5). The solid line is very different from the seasonal component of the SD (left-hand side of Equation 5). The result is an increase in the quarter-to-quarter change of the discrepancy instead of a significant decrease. Therefore, the analysis of the economic growth and its components is severely hampered.

In a preliminary study, we tried to reduce the inconsistencies by improving the settings in X-13ARIMA-SEATS. We found that a reduction is possible, but reducing them to an

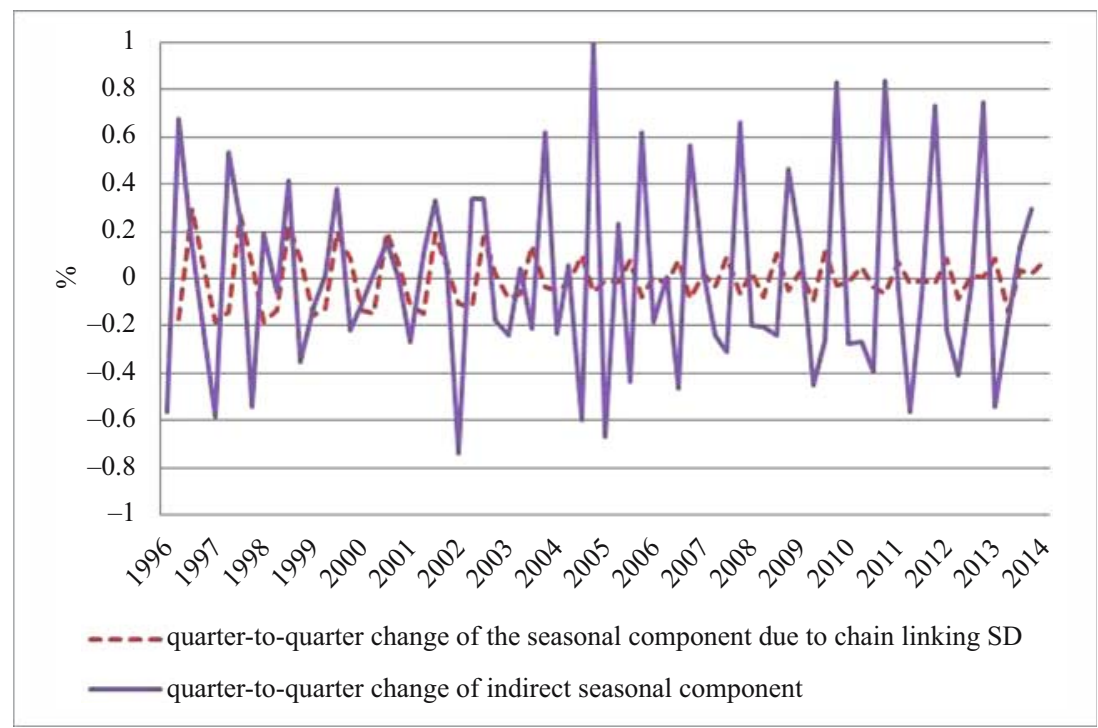

Fig. 3. Seasonal component of the discrepancies arising from chain linking calculated directly and indirectly with conventional univariate seasonal adjustment. 
acceptable level or even eliminating them completely and at the same time maintaining sufficient quality of the seasonal and calendar correction does not seem to be possible in this application. The lessons we learned in this study are nevertheless useful for the solution we found, described below. The most important lessons are:

- The largest part of the discrepancies is caused by the pretreatment, especially the treatment of outliers.

- An additive adjustment approach for all series helps to reduce the discrepancies compared to a multiplicative adjustment. Although a multiplicative adjustment is preferred for the series of export, GDP and consumption of households, an additive adjustment is applied. The quality under additive correction is acceptable also for the series where multiplicative correction is preferred. The differences between these approaches are small in practice, at least for the GDP in the Dutch situation.

- The best results could be obtained by an equivalent approach, that is, using the same settings (ARIMA models, filters), modelling outliers in the same periods, additive adjustment for all series, and using the same set of auxiliary variables for all series. This results in a less optimal adjustment according to the Q- and M-diagnostics of X-13ARIMA-SEATS.

The discrepancies are still unacceptably large, even under an optimally chosen equivalent approach and therefore not further implemented in the production of official releases. These findings have led to the conclusion that a multivariate approach is needed in order to reach all three objectives, that is, reducing discrepancies while maintaining univariate quality and avoiding large revisions. This approach is presented in the next section.

\section{Multivariate Structural Time Series Model}

In this section, a multivariate structural time series modelling approach is developed for the purpose of estimating seasonal effects for an aggregated series and its breakdown in $K$ series in a consistent way. With a structural time series model (STM), a series is decomposed in a trend component, seasonal component, regression components and an irregular component. The model can be extended with other components as cyclic components, or with ARMA components to model autocorrelation beyond these structural components, but this is not applied in the present article. For each component, an appropriate stochastic model is assumed which allows the trend, seasonal, and regression coefficients to be time-dependent. See Harvey (1989) and Durbin and Koopman (2012) for an extensive treatment of structural time series modelling. In multivariate STMs, two or more series are modelled simultaneously, which allows modelling cross-sectional dependency between these series.

\subsection{Consistent Seasonal Adjustment with a Multivariate STM}

We developed a multivariate STM for quarterly GDP, broken down into a hierarchy according to either the expenditure approach or production approach. Either hierarchy contains multiple levels (see Appendix (Section 6) for the breakdown of expenditures). At every level, there is a statistical discrepancy before seasonal and calendar adjustment (but only if measured in constant prices). The breakdown of GDP into seven subseries 
(including the statistical discrepancy) is defined in Equation (1). The time series modelling approach outlined in this section can be applied to each hierarchy of GDP (see Appendix (Section 6) for details). It is important that the consistency between all hierarchical levels of GDP is maintained. This is done repeatedly in a top-down approach. In each hierarchy, restrictions are imposed that ensure that for every subsequent level, all time series components are benchmarked to estimates of the aggregate at the higher level.

Let $y_{t+}$ be the GDP as measured on a quarterly basis. In the first step, the following univariate STM is estimated:

$$
y_{t+}=L_{t}+S_{t}+\alpha \Delta_{t}^{O}+\beta_{t} x_{t}+\lambda \Delta_{t}^{L}+\gamma_{t} \Delta_{t}^{S}+e_{t} .
$$

The trend-cycle $L_{t}$ is modelled according to the smooth trend model and the seasonal pattern $S_{t}$ is modelled using a trigonometric model (Durbin and Koopman, 2012, chap. 3; and supplemental file of this article). Furthermore $\Delta_{t}^{O}$ is a dummy variable, indicating the period in which an additive outlier occurs, that is,

$$
\Delta_{t}^{O}= \begin{cases}1 & \text { for the period } t \text { where an outlier occurs } \\ 0 & \text { for all other periods }\end{cases}
$$

and $\alpha$ denotes the corresponding time-invariant regression coefficient measuring the magnitude of the outlier. In (6), $\Delta_{t}^{L}$ is a dummy variable indicating the period in which a level shift occurs, that is,

$$
\Delta_{t}^{L}=\left\{\begin{array}{l}
0 \text { for all } t \text { before the period in which a level shift occurs } \\
1 \text { for all } t \text { from (and including) the period in which a level shift occurs }
\end{array}\right.
$$

and $\lambda$ denotes a time-invariant regression coefficient measuring the size of the level shift. A break in the seasonal pattern is modelled with a similar intervention variable:

$$
\Delta_{t}^{S}=\left\{\begin{array}{l}
0 \text { for all } t \text { before the period in which a seasonal break occurs } \\
1 \text { for all } t \text { from (and including) the period of the seasonal break }
\end{array}\right.
$$

The magnitude of the seasonal break is measured by $\gamma_{t}$, which is defined as a timeinvariant trigonometric seasonal model. This implies that all four quarters have their own break (adding up to zero) which are time invariant. Furthermore $x_{t}$ denotes the number of working days that is used to model calendar effects in period $t$, and $\beta_{t}$ denotes the corresponding time-dependent regression coefficient modelled as a random walk (Durbin and Koopman 2012, chap. 6). The regression coefficient is allowed to vary over time, since GDP generally increases over time and therefore, also, the size of the working day effect. Finally $e_{t}$ is a disturbance term for any unexplained variations.

In the general case, multiple additive outliers, level shifts and seasonal breaks are possible, and multiple auxiliary variables may be useful. Then, Equation (6) can be adapted in a straightforward way.

Based on (6), smoothed estimates for total GDP (or the aggregated series of another hierarchy) are obtained. In a second step, the $K$ subseries (without the aggregated series), 
represented by a $K$-dimensional vector $\left(y_{t 1}, \ldots, y_{t k}, \ldots, y_{t K}\right)^{\prime}$, are modelled by a $K$-dimensional multivariate STM:

$$
y_{t k}=L_{t k}+S_{t k}+\alpha_{k} \Delta_{t}^{O}+\beta_{t k} x_{t}+\lambda_{k} \Delta_{t}^{L}+\gamma_{t k} \Delta_{t}^{S}+e_{t k}, \quad k=1 \ldots K
$$

The various components in (10) are defined similarly as in Equation (6), but now, for each series $k=1, \ldots K$, separately. Outliers, level shifts and seasonal breaks may be zero for some series if the analysis shows that they do not occur in a particular series.

To avoid an increase of discrepancies due to seasonal and calendar adjustment, several constraints are imposed on the time series components. These constraints ensure that, for each of these components at each point in time, the value for the aggregate series is exactly equal to the sum of the values of the underlying subseries. Therefore, Equation (10) is applied with the restriction that the sum of the various state variables equals the smoothed values obtained in (6). This is done using the benchmark procedure proposed by Doran (1992). We have constraints for the following components:

- The trend components:

$$
L_{t+}=\sum_{k=1}^{K} L_{t k}
$$

- The regression coefficients for the working day effects:

$$
\beta_{t+}=\sum_{k=1}^{K} \beta_{t k}
$$

- The seasonal components:

$$
S_{t+}=\sum_{k=1}^{K} S_{t k}
$$

- Outliers:

$$
\alpha_{+}=\sum_{k=1}^{K} \alpha_{t k}
$$

- Level shifts:

$$
\lambda_{+}=\sum_{k=1}^{K} \lambda_{t k}
$$

- Seasonal breaks:

$$
\gamma_{t+}=\sum_{k=1}^{K} \gamma_{t k}
$$

With the initially intended seasonal adjustment method, the consistent estimates for the seasonal components $S_{t k}$, seasonal breaks $\gamma_{t k}$, and calendar effects $\beta_{t k} x_{t}$ are used for 
seasonal and calendar adjustment. Since these estimates obey restrictions in Equations (13), (16), and (12), the adjustment procedure does not increase discrepancies.

In order to estimate the multivariate STM described above, it is written in state space form. The state space representation of Equations (6) and (10) is given in the supplement of this article. Next, the Kalman filter is used to obtain optimal estimates for all state variables (see Durbin and Koopman 2012; Harvey 1989). The Kalman filter is a recursive procedure to obtain optimal estimates for the state vector at time $t$ based on the data up to and including time period $t$. These estimates are referred to as the filtered estimates. The filtered estimates of past state vectors can be updated, if new data become available. This procedure is referred to as smoothing. Several smoothing algorithms are available in the literature. In this article, the fixed interval smoother is applied, which is a broadly applied smoothing algorithm, and these estimates are referred to as the smoothed estimates. The Kalman filter assumes that the hyperparameters are known, which is generally not the case. Therefore, they are estimated with a maximum likelihood procedure. Finally, we apply diffuse initialization of the Kalman filter for all the state variables.

These models are analyzed with a program that was developed in Oxmetrics (Doornik 2009), using the procedures of Ssfpack 3.0 (Koopman et al. 1999, 2008). Ssfpack is a library of subroutines developed for analyzing (multivariate) STMs. Standard model diagnostics summarized in Durbin and Koopman (2012, chap. 2) are applied to evaluate whether the innovations meet the assumption that they are normally and independently distributed.

Several forms of Model (10) are applied to total GDP and its breakdown in seven series defined by (1). The main differences are in the covariance structures assumed for the disturbance terms of the trend, seasonal component and regression coefficients, varying from full covariance matrices, diagonal matrices and diagonal covariance matrices with equal variances for several series. A general result obtained with these models is that the estimated components, especially the seasonal component, were volatile, and subject to large revisions when data points were added to the time series. From a practical perspective, this is not desirable. In many cases, there are problems with the numerical optimization procedure applied to find a maximum of the likelihood function. To circumvent these issues, an alternative three-step approach is developed to solve problems with large discrepancies. This new approach consists of pretreatment based on a multivariate STM that ensures that the components that contributed most to the discrepancies are consistent. The seasonal adjustment itself is carried out with X-13ARIMA-SEATS. A seasonal adjustment approach based on a multivariate STM is left as a topic for further research.

\subsection{Multivariate STM for Pretreatment}

The following procedure, which combines the advantages of a multivariate approach and the robustness of conventional univariate seasonal adjustment with X-13ARIMASEATS, is proposed as a practical solution for handling discrepancies. In a first step, the univariate STM (6) is applied to the aggregated series. Then, the multivariate STM (10) is applied to the $K$ subseries with restrictions (12), (14), (15), (16), and diagonal covariance matrices for the disturbances of the trends and the seasonal components. 
These results are used to remove additive outliers, level shifts, seasonal breaks, and calendar effects from the series. In a second step, X-13ARIMA-SEATS via JDemetra+ (Grudkowska 2015) is used for the extrapolation of the series and to obtain seasonally adjusted series by applying trend and seasonal filters. After that, the additive outliers, level shifts, and calendar effects removed in the first step are reintroduced in the series. In a third step, multivariate benchmarking is applied to eliminate any remaining discrepancies.

The final seasonally adjusted series are the seasonally adjusted series plus the level shifts and additive outliers removed in the pretreatment. Seasonal breaks are not added back to the series, because the purpose of seasonal adjustment is to remove seasonal patterns.

Effectively, this means that the multivariate model is only used for pretreatment, comparable to this step in X-13ARIMA-SEATS (except for the extrapolation, which is done by X-13ARIMA-SEATS in our approach). The advantage of carrying out this pretreatment with a multivariate STM is that calendar effects, additive outliers, level shifts and seasonal breaks are fully consistent between the subseries and the aggregate. To this end, we used the same regressors for all series, and outliers were modelled consistently, as described in the next subsection. Since these components obey restrictions (12), (14), (15) and (16), we obtained a close-to-optimal result, which is more stable than the approach based on estimating consistent seasonal effects with the multivariate STM. An empirical result of this approach is that if pretreatment yields small discrepancies, then most likely the final seasonal adjustment will not increase these discrepancies by much, see Subsection 2.2.

The increase in discrepancies due to univariate seasonal adjustment with X-13ARIMASEATS is minimized if the same filter length is used for all series. We chose a short seasonal filter, since the seasonal pattern changes quite rapidly. This had only a slight effect on the quality of univariate seasonal adjustment. The ARIMA model used for extrapolation was determined for the aggregate and applied for each series in the breakdown. This procedure results in seasonally adjusted series that have only very small discrepancies. In order to remove these, a multivariate benchmarking procedure was applied.

\subsection{Consistent Outlier Detection}

Three types of outliers are distinguished: additive outliers, level shifts and seasonal breaks. As outliers can be much larger than the seasonal and calendar effects, their influence can be very large. Detecting and modelling them is crucial for achieving good seasonal adjustment. In some cases, more than one outlier is needed to model the economic events in a short period of time. On the other hand, it is difficult to find the optimal combination of outliers and avoid overfitting of the series. An observation is that not using the right combination of outliers results in a serious deterioration of quality diagnostics of X-13ARIMA-SEATS. Another consequence is that the estimates of all state variables can become unstable.

For every significant outlier in one of the series, there must be a counterpart in one or more of the other series to achieve consistency. These counterparts are not necessarily 
significant, and therefore difficult to detect. Sometimes there are substantive economic explanations for the occurrence of outliers that are helpful in choosing the type of outlier, timing, the counterparts and in some cases, even the size of the outliers. When the size of an outlier can be determined from statistical/economic analysis, the outlier can be manually removed from the series and does not need to be modelled in the multivariate STM. This results in a more parsimonious model, which is therefore preferred. When the size of the outliers cannot be determined, we model the outliers in the STM.

For around $50 \%$ of the outliers, this additional information is not available and outliers are detected in a model selection process. Outliers are detected using the automatic detection in the pretreatment phase of X-13ARIMA-SEATS. This method is based on a RegARIMA model. These outliers are modelled in the multivariate STM. Additional outliers are detected by considering the residuals of the STM using a disturbance smoother (Harvey and Koopman 1992). For all residuals with a $t$-value larger than 2.5, an outlier and its counterparts are added to the set.

The set of outliers that is modelled explicitly in the pretreatment phase is removed from the series. The pretreated series become the input for the seasonal adjustment phase. Nevertheless, the seasonal adjustment phase in X-13ARIMA-SEATS can detect additional outliers. As these decisions are made for each series separately, they will again lead to statistical discrepancies. Therefore, we must model these events with additional outliers in the pretreatment phase. In order to reduce the number of outliers detected in the seasonal adjustment phase, we increased the critical value that controls whether an observation is classified as an outlier. All outliers that were detected above this level were added to the set of outliers modelled in the pretreatment phase. This process is iterated until no new outliers are detected.

\section{Results}

In this section, we apply the seasonal and calendar adjustment approach obtained with standard X-13ARIMA-SEATS (old method) and the improved method proposed in Section 3 to the cycle of releases of the quarterly GDP and its breakdown in components according to (1) in an annual estimation cycle and compare the results obtained under both approaches. The old method refers to the application of X-13ARIMA-SEATS with settings that conform to Eurostat guidelines (Eurostat 2015), as applied in the production process. This means that for part of the series the logarithmic transformation is applied. The set of outliers under the old method is different from the set under the improved methods.

The quarterly GDP figures are produced twice: 45 days after the end of a quarter, a flash estimate is published. Then, 85 days after the end of the quarter a new regular estimate is published, based on more complete data sources. When the regular estimate of the fourth quarter is published in March, the figures for the first three quarters are revised again.

The quarterly figures are revised three more times after that: for each new annual estimate, the quarterly figures are adapted such that the four quarters add up to the new annual figure. This happens for the first time in June and for the second time one year later, when the final annual figures are published. Finally, one year after this, the quarterly figures are revised one more time without changing the annual results. Furthermore, every 
time a new quarter is added, the seasonal adjustment procedure is applied to the entire time series, potentially affecting all quarters. However, normally, revisions of seasonal adjustments to earlier figures are small. Since 2016, the figures are revised twice because the process has been accelerated. This implies that the second revision is the final one, where only quarterly figures are adjusted.

This means that once a year, at the time that the regular estimate for the first quarter (1r) is made, large changes are made to the (unadjusted) time series. Therefore, it is necessary to derive new settings for the seasonal and calendar adjustment at this point in time every year. The annual estimation cycle starts with the regular estimate for the first quarter, and the derived settings are then used for all subsequent estimates of the annual estimation cycle. The 1r estimate is followed by the first (flash) estimate of the second quarter (2f) and the second estimate of the second quarter (2r). This scheme is continued until the second estimate of the fourth quarter (4r). The first estimate of the first quarter of the current year comes before the large updates of $1 \mathrm{r}$ and therefore, is also part of the same cycle. It is called 5f, to emphasize that the settings of the previous year are applied.

Below, the old method and the improved method (without the final benchmarking step) are compared according to the three quality criteria described in Section 1:

- The statistical discrepancy between the seasonally adjusted GDP and its components.

- The standard quality diagnostics of X-13ARIMA-SEATS: M1 to M11 and Q.

- The revisions of the published results between the subsequent estimations.

We use data from the time period 1996-2014 for the computations in this section.

\subsection{The Statistical Discrepancy due to Seasonal and Calendar Adjustment}

In this subsection, we discuss the discrepancies added by the seasonal and calendar adjustment process. This process estimates the seasonal components of each of the series. In both the old approach and the improved approach, the estimated seasonal components of all subseries do not add up to the seasonal component of GDP, and result in a residual:

$$
\Delta=S_{B 1 G}+S_{P 7}-S_{P 6}-S_{P 3_{S 1 A}}-S_{P 3_{S 13}}-S_{P 51 G}-S_{P 5 M}-S_{S D}
$$

In Table 1, we compare the added statistical discrepancy of the old method and the improved method, by taking the relative added statistical discrepancy computed as a

Table 1. Average and maximum absolute discrepancies due to seasonal adjustment for the year 2014.

\begin{tabular}{|c|c|c|c|c|}
\hline & \multicolumn{2}{|c|}{ Old method } & \multicolumn{2}{|c|}{ Improved method } \\
\hline & Avg \% & $\operatorname{Max} \%$ & Avg \% & $\operatorname{Max} \%$ \\
\hline $1 \mathrm{r}$ & 0.331 & 1.01 & 0.001 & 0.01 \\
\hline $2 \mathrm{f}$ & 0.329 & 1.00 & 0.001 & 0.01 \\
\hline $2 r$ & 0.321 & 1.00 & 0.001 & 0.01 \\
\hline $3 f$ & 0.321 & 0.99 & 0.001 & 0.01 \\
\hline $3 r$ & 0.320 & 0.99 & 0.001 & 0.01 \\
\hline $4 \mathrm{f}$ & 0.322 & 0.98 & 0.001 & 0.01 \\
\hline $4 r$ & 0.330 & 0.99 & 0.001 & 0.01 \\
\hline $5 f$ & 0.313 & 0.99 & 0.000 & 0.01 \\
\hline
\end{tabular}


percentage change from seasonally adjusted GDP:

$$
\left|\frac{\Delta}{B 1 G^{S A}}\right| * 100 \%
$$

Table 1 presents the average and maximum of this difference over the entire time series.

Table 1 shows that a significant reduction in statistical discrepancy due to seasonal and calendar adjustment can be achieved by using the improved method. With the old method, interpretation of GDP growth was, on average, hampered by the discrepancy by $0.3 \%$, with a maximum of $1 \%$, while, with the new method, the disturbance is negligible.

\subsection{The Standard Diagnostics of X-13ARIMA-SEATS}

Software of the X-11-family summarizes the quality of the seasonal and calendar adjustment with M1 to M11 and a Q-diagnostics. These diagnostics value different aspects of the seasonally adjusted series. For the meaning of the values, see the supplemental file or Ladiray and Quennville (2001). These statistics vary between 0 and 3. Values smaller than 1 are to be preferred, however are not always achievable due to characteristics of the series. The lower the value, the better. Tables 2 and 3 present the diagnostics of the seasonal adjustment with the old method and the improved method of estimate 1r.

In Table 2, eight diagnostics are between 1 and 2 and two of them are above 2. This shows that the quality of the seasonal and calendar adjustment is not always satisfactory, but further substantial improvements are not possible using traditional methods. With the new method, the results have improved to six diagnostics above 1 and none above 2. On the other hand, the new method has fewer quality diagnostics with very small values. On average, the quality improves slightly. This is due to the improved analysis of the outliers. The multivariate pretreatment of the new method results in fewer quality diagnostics with very high and very low values.

Table 2. Quality of seasonally adjusted estimate $1 r$ (for the year 2014) with old method (values $>1$ are bold).

\begin{tabular}{lccccccc}
\hline & $\begin{array}{c}\text { Import } \\
(P 7)\end{array}$ & $\begin{array}{c}\text { Consumption } \\
\text { HH } \\
\left(P 3_{S 1 A}\right)\end{array}$ & $\begin{array}{c}\text { Consumption } \\
\text { govern } \\
\left(P 3_{S 13}\right)\end{array}$ & $\begin{array}{c}\text { Cap. form. } \\
(P 51 G)\end{array}$ & $\begin{array}{c}\text { Stocks } \\
(P 5 M)\end{array}$ & $\begin{array}{c}\text { Export } \\
(P 6)\end{array}$ & $\begin{array}{c}\text { GDP } \\
(B 1 G)\end{array}$ \\
\hline M1 & 0.49 & 0.42 & 0.00 & 0.09 & 0.33 & 0.10 & 0.05 \\
M2 & 0.64 & 0.00 & 0.00 & 0.06 & 0.19 & 0.05 & 0.02 \\
M3 & 0.13 & 0.00 & 0.00 & 0.31 & 0.39 & 0.00 & 0.00 \\
M4 & 0.18 & $\mathbf{1 . 1 6}$ & 0.95 & $\mathbf{1 . 0 5}$ & 0.84 & 0.84 & 0.84 \\
M5 & 0.24 & 0.20 & 0.20 & 0.20 & 0.41 & 0.20 & 0.20 \\
M6 & 0.12 & 0.44 & $\mathbf{1 . 0 0}$ & 0.16 & 0.64 & 0.52 & 0.22 \\
M7 & 0.38 & 0.39 & 0.16 & 0.13 & 0.32 & 0.24 & 0.06 \\
M8 & 0.91 & 0.71 & 0.51 & 0.53 & $\mathbf{1 . 1 2}$ & 0.60 & 0.17 \\
M9 & 0.54 & 0.64 & 0.26 & 0.14 & 0.67 & 0.34 & 0.06 \\
M10 & $\mathbf{1 . 3 3}$ & $\mathbf{1 . 0 4}$ & 0.38 & 0.54 & $\mathbf{2 . 3 0}$ & 0.98 & 0.17 \\
M11 & $\mathbf{1 . 3 0}$ & $\mathbf{1 . 0 2}$ & 0.22 & 0.44 & $\mathbf{2 . 3 0}$ & 0.97 & 0.17 \\
Q & 0.46 & 0.45 & 0.22 & 0.29 & 0.64 & 0.34 & 0.16 \\
\hline
\end{tabular}


Table 3. Quality of seasonally adjusted estimate $1 r$ (for the year 2014) with improved method.

\begin{tabular}{lccccccc}
\hline & $\begin{array}{c}\text { Import } \\
(P 7)\end{array}$ & $\begin{array}{c}\text { Consumption } \\
\text { HH } \\
\left(P 3_{S 1 A}\right)\end{array}$ & $\begin{array}{c}\text { Consumption } \\
\text { govern } \\
\left(P 3_{S 13}\right)\end{array}$ & $\begin{array}{c}\text { Cap. form. } \\
(P 51 G)\end{array}$ & $\begin{array}{c}\text { Stocks } \\
(P 5 M)\end{array}$ & $\begin{array}{c}\text { Export } \\
(P 6)\end{array}$ & $\begin{array}{c}\text { GDP } \\
(B 1 G)\end{array}$ \\
\hline M1 & 0.33 & 0.61 & 0.02 & 0.27 & 0.73 & 0.18 & 0.04 \\
M2 & 0.17 & 0.02 & 0.03 & 0.18 & 0.55 & 0.08 & 0.02 \\
M3 & 0.30 & 0.17 & 0.27 & 0.86 & $\mathbf{1 . 1 0}$ & 0.03 & 0.00 \\
M4 & 0.95 & 0.51 & 0.51 & 0.40 & 0.18 & $\mathbf{1 . 0 5}$ & 0.40 \\
M5 & 0.20 & 0.20 & 0.20 & 0.60 & 0.95 & 0.20 & 0.20 \\
M6 & 0.08 & $\mathbf{1 . 3 2}$ & 0.74 & $\mathbf{1 . 4 1}$ & 0.81 & 0.31 & 0.16 \\
M7 & 0.30 & 0.19 & 0.24 & 0.14 & 0.19 & 0.18 & 0.10 \\
M8 & 0.82 & 0.72 & 0.51 & 0.68 & $\mathbf{1 . 0 2}$ & 0.60 & 0.33 \\
M9 & 0.45 & 0.13 & 0.26 & 0.19 & 0.21 & 0.25 & 0.22 \\
M10 & 0.64 & 0.52 & 0.36 & 0.78 & $\mathbf{1 . 2 3}$ & 0.38 & 0.22 \\
M11 & 0.32 & 0.40 & 0.24 & 0.17 & 0.48 & 0.16 & 0.12 \\
Q & 0.40 & 0.30 & 0.24 & 0.39 & 0.61 & 0.28 & 0.14 \\
\hline
\end{tabular}

The quality of GDP is almost the same as before, despite the fact that in the multivariate approach the excellent univariate seasonal and calendar adjustment is slightly disturbed by the other series. The quality of the gross fixed capital formation $(P 51 G)$ deteriorates because M5 worsens due to the larger level shift in 2009-Q1 (resulting in less trend) in the multivariate case compared to the univariate case. M1 and M2 deteriorate under the new method because this series contains a larger irregular component. This is the result of using larger critical values for detecting outliers in X-13ARIMA-SEATS in the seasonal adjustment phase. This also affects M6 and M8, resulting in larger arbitrary changes of the seasonal component. The results for M9 to M11 are greatly improved. This is caused by the modelling of the seasonal outliers. In both methods, the sum of the four quarters of a seasonal outlier adds up to zero. However, with the improved method, a seasonal outlier has a different magnitude in every quarter, while with the old method, the outlier is determined in one quarter and the three other quarters have a third of its opposite magnitude. A disadvantage of the improved method is that it uses three quarters in the time series to determine the outlier, while the old method only uses one degree of freedom.

Remarkably large differences in diagnostics are found for M4 for the import $(P 7)$ and the export (P6): both deteriorate. Further analysis showed that M4 could be improved by adding an extra seasonal break in 2003, which becomes more pronounced due to the seasonal break of 2008. However, this was unknown during the implementation of the new method for the seasonal adjustment of the Dutch quarterly national accounts.

Table 4 presents the difference in overall quality (as measured by the Q-diagnostic) between the two methods for all eight estimates. Negative values (in bold) relate to an improvement by using the new method, positive values relate to a deterioration. Both methods display an almost constant difference in quality during the annual cycle. 
Table 4. Difference in Q-diagnostic between old and new method for seasonal adjustment, for the year 2014.

\begin{tabular}{|c|c|c|c|c|c|c|c|}
\hline & $\begin{array}{c}\text { Import } \\
(P 7)\end{array}$ & $\begin{array}{c}\text { Consumption } \\
\mathrm{HH} \\
\left(P 3_{S 1 A}\right)\end{array}$ & $\begin{array}{c}\text { Consumption } \\
\text { govern } \\
\left(P 3_{S 13}\right)\end{array}$ & $\begin{array}{l}\text { Stocks } \\
(P 5 M)\end{array}$ & $\begin{array}{l}\text { Cap. form. } \\
(P 51 G)\end{array}$ & $\begin{array}{c}\text { Export } \\
(P 6)\end{array}$ & $\begin{array}{c}\text { GDP } \\
(B 1 G)\end{array}$ \\
\hline $1 \mathrm{r}$ & -0.06 & -0.15 & 0.02 & 0.10 & -0.02 & $-\mathbf{0 . 0 7}$ & $-\mathbf{0 . 0 1}$ \\
\hline $2 f$ & $-\mathbf{0 . 0 8}$ & $-\mathbf{0 . 1 0}$ & 0.03 & 0.10 & $-\mathbf{0 . 0 7}$ & $-\mathbf{0 . 0 7}$ & -0.02 \\
\hline $2 r$ & $-\mathbf{0 . 0 7}$ & -0.10 & 0.03 & 0.10 & -0.07 & -0.06 & $-\mathbf{0 . 0 1}$ \\
\hline $3 \mathrm{f}$ & $-\mathbf{0 . 0 8}$ & -0.11 & 0.02 & 0.13 & -0.06 & $-\mathbf{0 . 0 7}$ & $-\mathbf{0 . 0 1}$ \\
\hline $3 r$ & -0.08 & -0.11 & 0.02 & 0.13 & -0.06 & $-\mathbf{0 . 0 7}$ & $-\mathbf{0 . 0 1}$ \\
\hline $4 \mathrm{f}$ & $-\mathbf{0 . 0 7}$ & -0.12 & 0.00 & 0.16 & -0.04 & -0.08 & $-\mathbf{0 . 0 1}$ \\
\hline $4 \mathrm{r}$ & -0.06 & -0.16 & 0.00 & 0.09 & $-\mathbf{0 . 0 3}$ & -0.08 & $-\mathbf{0 . 0 2}$ \\
\hline $5 f$ & -0.05 & -0.14 & 0.01 & 0.12 & $-\mathbf{0 . 0 3}$ & -0.06 & $-\mathbf{0 . 0 2}$ \\
\hline
\end{tabular}

\subsection{Revisions}

In this section, revisions of the quarter-to-quarter growth in \%-point are investigated under the old method and the improved method.

The quarter-to-quarter growth is defined as

$$
\hat{\theta}_{t}=\frac{y_{t}^{S A}-y_{t-1}^{S A}}{y_{t-1}^{S A}} \cdot 100 \%,
$$

where $y_{t}^{S A}$ denotes the seasonally adjusted figures. This is computed for the GDP and the variables of the breakdown.

The revisions are split into two types; the first are due to the updates from flash to regular estimate:

$$
R_{1}=\left(\sum_{t=2}^{4}\left|\hat{\theta}_{t \mid t}^{f}-\hat{\theta}_{t \mid t}^{r}\right|\right) .
$$

with $\hat{\theta}_{t \mid T}^{f}$ and $\hat{\theta}_{t \mid T}^{r}$ the flash and regular estimates of the growth rates, see formula (17), for quarter $t$ based on the time series up to and including quarter $T$.

Note that the first quarter $(t=1)$ is excluded in $R_{1}$ since in the regular estimate of this quarter, the information on an annual basis is added, which causes large revisions.

The second type of revisions is due to adding the flash estimate of a new quarter:

$$
R_{2}=\left(\sum_{t=1}^{4}\left|\hat{\theta}_{t \mid t}^{r}-\hat{\theta}_{t \mid t+1}^{f}\right|\right)
$$

The average revision of the last quarter is therefore:

$$
\frac{1}{7}\left(R_{1}+R_{2}\right)
$$

which is presented in Figure 4.

Similarly, Figure 5 presents the average absolute revisions of the quarter-to-quarter growth in \%-point over eight estimates (i.e., seven differences) over the last year 


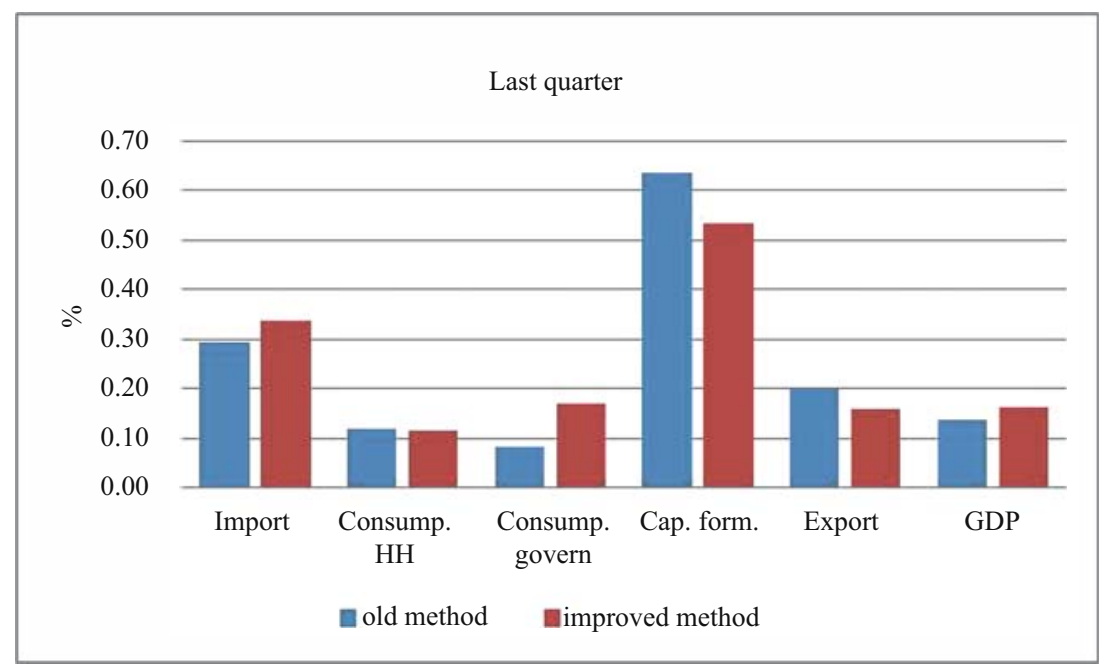

Fig. 4. Average revision of quarter-to-quarter growth in 2014 defined by equation (19).

averaged per quarter:

$$
\begin{gathered}
\frac{1}{28} \sum_{j=0}^{3}\left(R_{1 j}+R_{2 j}\right) \\
\text { with } \quad R_{1 j}=\left(\sum_{t=2}^{4}\left|\hat{\theta}_{t-j \mid t}^{f}-\hat{\theta}_{t-j \mid t}^{r}\right|\right), \mathrm{j}=0,1,2,3 \\
\text { and } \quad R_{2 j}=\left(\sum_{t=1}^{4}\left|\hat{\theta}_{t-j \mid t}^{r}-\hat{\theta}_{t-j \mid t+1}^{f}\right|\right), \mathrm{j}=0,1,2,3
\end{gathered}
$$

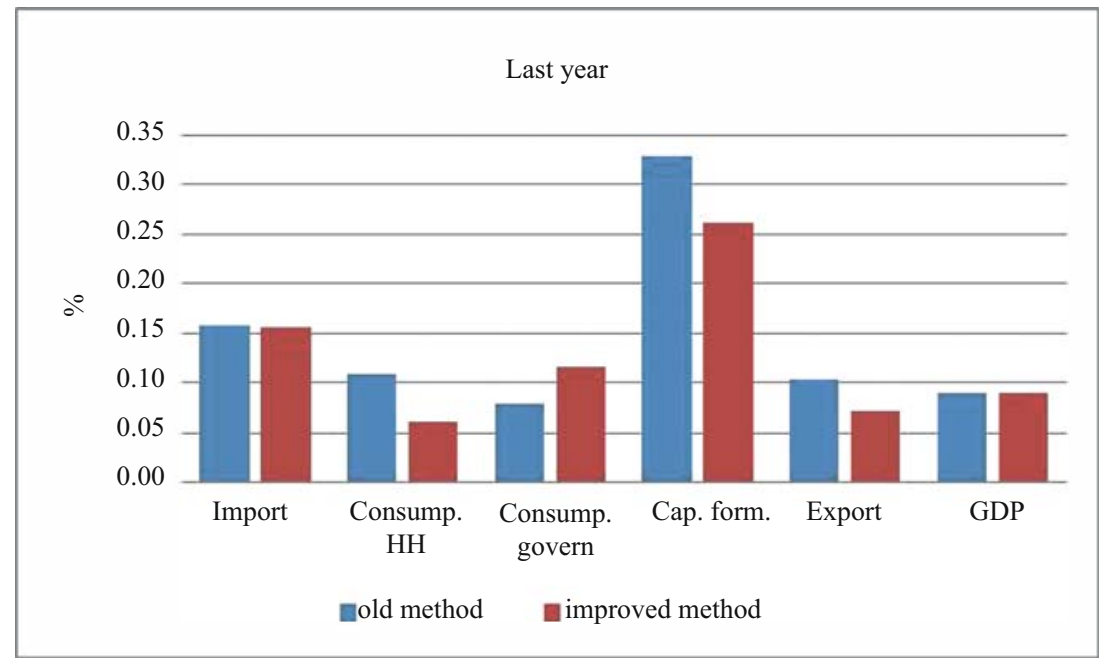

Fig. 5. Average revision of annual growth in 2014 defined by equation (20). 
Series SD (statistical discrepancy due to chain linking) and P5M (changes in stocks) are both fluctuating around zero. Therefore, both can have huge growths in \%-points because of small absolute values, resulting in huge revisions of the growth. As a consequence, they are left out of the analysis. The figures show that the size of the revisions of the old method and the improved method are almost equal. For consumption government a small deterioration is observed, but the revisions are still very small. The deterioration is caused by the time-varying seasonal pattern, which is picked up faster under the improved method due to the use of shorter seasonal filters. A reduction of the revisions was not expected in advance, as adding or changing observations at the end of the series gives new information about trend-cycle and seasonal component. Revisions are therefore inherent to seasonal and calendar adjustment.

\section{Conclusion}

Quarterly figures about GDP with a breakdown in $K$ underlying subseries for, for example, expenditures or industries, are produced by national statistical institutes to measure and analyze economic growth. Two factors are responsible for discrepancies between the sum of the underlying $K$ subseries and the total GDP. The first factor arises due to the process of chain linking, which means that series of volume growth rates are expressed in constant price levels. Since the annual changes of these price levels differ between the series, statistical discrepancies between the sum of the underlying series and total GDP arise. The first factor does not arise if the estimate is in current values. The second factor arises after adjusting for seasonal and calendar effects using the standard approach based on X-13ARIMA-SEATS. In the Netherlands, since 2009, the size of these discrepancies has often been larger than the growth rates of GDP itself and hampers the interpretation of these figures.

Several intuitive approaches to avoid discrepancies are available in the literature, such as the indirect approach and multivariate benchmarking. A major drawback of the first approach is that official figures about GDP are derived from the most detailed breakdown, which contains the largest fluctuations, while the most reliable estimates at the aggregated level are not used. Benchmarking is appropriate if the discrepancies are modest. In the Dutch application, the discrepancies are large, and benchmarking introduces a residual seasonal effect in the adjusted series.

In this article, an alternative approach based on a multivariate structural time series model is considered. The most intuitive approach is to construct a $K+1$ dimensional structural time series model for GDP and its breakdown in $K$ subseries. The model contains explicit constraints on the state variables to ensure that trend, seasonal effects, calendar effects and outliers in GDP are equal to the sum of the $K$ subseries of these components. In this way, available series are consistently modelled and a two-stage approach is avoided. Nevertheless, the results obtained with this approach are not satisfactory since the estimated seasonal effects are too volatile. Furthermore, we observed numerical problems with the maximum likelihood procedure for the hyperparameters and the revisions were too large. Solving these problems is left as a topic for further research. 
As an alternative, a multivariate structural time series model with consistency restrictions on the additive outliers, level breaks, seasonal breaks and calendar effects (derived from a univariate model applied to the aggregated series) is only used to eliminate these effects from the observed series. Subsequently, X-13ARIMA-SEATS is used for seasonal adjustment of all series. This reduces the inconsistencies remarkably. Finally, a multivariate benchmarking is applied to restore consistency in the adjusted series, and the additive outliers, level breaks, and calendar effects are added to the adjusted series. With this pretreatment approach, a significant reduction of the statistical discrepancies is achieved, whereas the quality of the adjustment in terms of the standard X-13ARIMA-SEATS quality measures is maintained or even improved for some series. In June 2015, this approach was implemented in the production of Dutch official statistics on economic growth.

When the numerical problems with the complete multivariate approach can be solved, comparing results of both approaches can give some insights into the influence of the approaches on the estimates.

The approach considered in this article is generic and applies to many other applications at national statistical institutes. Therefore, it is worthwhile to further improve the $K+1$ dimensional structural time series model, where consistent seasonal effects are directly estimated with the structural time series model.

\section{Appendix: Detailed breakdown of GDP}

Figure 6 summarizes the breakdown of GDP according to the expenditure approach. The official tables published by Statistics Netherlands are actually more detailed. Not presented here is the further breakdown of gross fixed capital formation. Each hierarchy of this breakdown is consistently corrected for seasonal and calendar effect using the top-down approach described in Subsection 3.2.

In each branch, there is a time series marked $S D x$. These are the discrepancies arising from chain linking. They only occur in constant price data. The figure illustrates that the breakdown comprises GDP itself and 20 subseries, complemented by nine different series for the discrepancies arising from chain linking (one for each branch of the tree). A similar breakdown tree of GDP is used for value added by industry (the production approach). This tree consists of six branches, and comprises 20 subseries for branches of industry and, of course, six series for discrepancies arising from chain linking. 


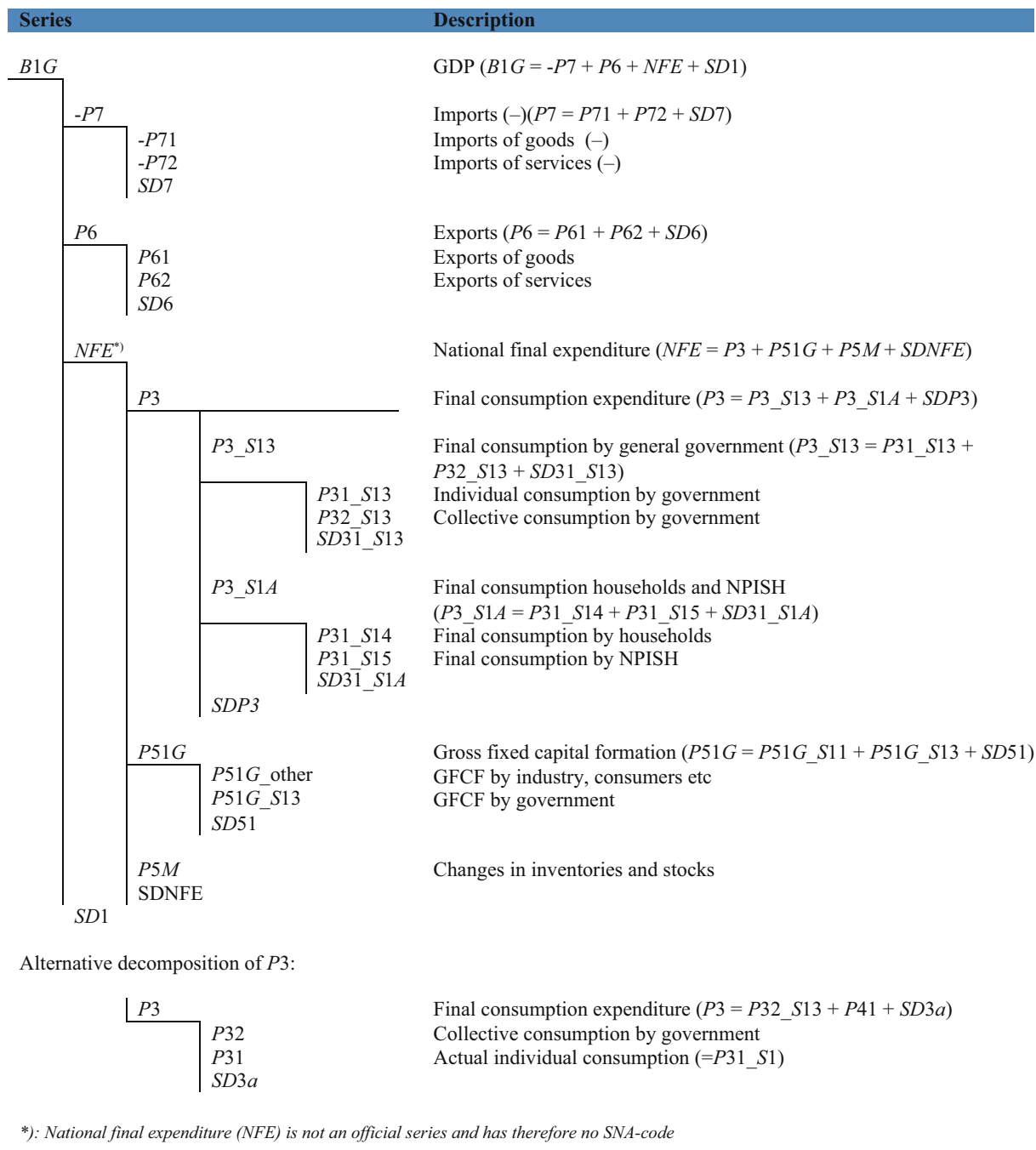

Fig. 6. Breakdown of GDP in components.

\section{References}

Bloem, A., R. Dippelsman, and N. Maehle. 2001. Quarterly National Accounts Manual-Concepts, Data sources and Compilation. Washington D.C. IMF. Available at: https://www.imf.org/external/pubs/ft/qna/2000/Textbook/ (accessed January 2019).

Di Fonzo, T. and M. Marini. 2011. "Simultaneous and Two-step Reconciliation of Systems of Time Series: Methodological and Practical Issues." Journal of the Royal Statistical Society C (Applied Statistics) 60. Part 2, 143-164. Doi: https://doi.org/ 10.1111/j.1467-9876.2010.00733.x.

Doornik, J.A. 2009. An Object-oriented Matrix Programming Language Ox 6. London: Timberlake Consultants Press. 
Doran, H.E. 1992. "Constraining Kalman Filter and Smoothing Estimates to Satisfy Time Varying Restrictions." Review in Economics and Statistics 74: 568-572. Doi: https://doi.org/10.2307/2109505.

Durbin, J. and S.J. Koopman. 2012. Time Series Analysis By State Space Methods (Second Edition). Oxford: Oxford University Press.

Eurostat. 2015. ESS Guidelines on Seasonal Adjustment (2015 Edition). Luxembourg: European Union. Available at: https://ec.europa.eu/eurostat/cros/content/ess-guidelinesseasonal-adjustment-2015-edition_en (accessed January 2019).

Grudkowska, S. 2015. JDemetra and User Guide. Warsaw: National Bank of Poland, Department of Statistics. Available at: https://ec.europa.eu/eurostat/cros/system/files/ jdemetra_user_guide.pdf (accessed January 2019).

Harvey, A.C. 1989. Forecasting, Structural Time Series Models and the Kalman Filter. Cambridge: Cambridge University Press.

Harvey, J.A. and S.J. Koopman. 1992. "Diagnostic Checking of Unobserved Components Time Series Models.” Journal of Business and Economic Statistics 10: 377-389. Doi: https://doi.org/10.1080/07350015.1992.10509913.

Koopman, S.J., N. Shephard, and J.A. Doornik. 1999. "Statistical Algorithms for Models in State Space Form Using Ssfpack 2.2." Econometrics Journal 2: 107-160. Doi: https://doi.org/10.1111/1368-423X.00023.

Koopman, S.J., N. Shephard, and J.A. Doornik. 2008. SsfPack 3.0: Statistical Algorithms for Models in State Space Form. London: Timberlake Consultants Press.

Ladiray, D. and B. Quenneville. 2001. Seasonal Adjustment with the X-11 Method. New York: Springer-Verlag.

Quenneville, B. and S. Fortier. 2006. "Balancing Seasonally Adjusted Series as a Complement to the Direct and Indirect Approaches to Seasonal Adjustment." Proc. Bus. Econ. Statist. Sect. Am. Statist. Ass.: 1118-1125.

U.S. Census Bureau. 2015. X-13ARIMA-SEATS Reference Manual, Version 1.1. Washington, D.C: U.S. Census Bureau. Available at: https://www.census.gov/ts/x13as/ docX13AS.pdf (accessed January 2019).

Received July 2017

Revised March 2018

Accepted August 2018 